\title{
XMM-Newton observation of a spectral state transition in the peculiar radio/X-ray/ $\gamma$-ray source LS I +61 303*
}

\author{
L. Sidoli ${ }^{1}$, A. Pellizzoni ${ }^{1}$, S. Vercellone ${ }^{1}$, M. Moroni ${ }^{1}$, S. Mereghetti ${ }^{1}$, and M. Tavani ${ }^{2,3,4}$ \\ 1 Istituto di Astrofisica Spaziale e Fisica Cosmica, Sezione di Milano, INAF-IASF, Milano 20133, Italy \\ e-mail: sidoli@iasf-milano.inaf.it \\ 2 CIFS, Torino 10133, Italy \\ 3 Istituto di Astrofisica Spaziale e Fisica Cosmica, Sezione di Roma, INAF-IASF, Roma 00133, Italy \\ 4 Dipartimento di Fisica, Università di Tor Vergata, Roma 00133, Italy
}

Received 29 June 2006 / Accepted 24 August 2006

ABSTRACT

\begin{abstract}
We report the results of XMM-Newton and BeppoSAX observations of the radio and X-ray emitting star LS I +61 303, likely associated with the gamma-ray source 2CG 135+01 and recently detected also at TeV energies. The data include a long XMM-Newton pointing carried out in January 2005, which provides the deepest look ever obtained for this object in the $0.3-12 \mathrm{keV}$ range. During this observation the source flux decreased from a high level of $\sim 13 \times 10^{-12} \mathrm{erg} \mathrm{cm}^{-2} \mathrm{~s}^{-1}$ to $4 \times 10^{-12} \mathrm{erg} \mathrm{cm}^{-2} \mathrm{~s}^{-1}$ within $2-3 \mathrm{~h}$. This flux range is the same seen in shorter and less sensitive observations carried out in the past, but the new data show for the first time that transitions between the two levels can occur on short time scales. The flux decrease was accompanied by a significant softening of the spectrum, which is well described by a power law with photon index changing from $1.62 \pm 0.01$ to $1.83 \pm 0.01$. A correlation between hardness and intensity is also found when comparing different short observations spanning almost 10 years and covering various orbital phases. LS I +61 303 was detected in the 15-70 keV range with the PDS instrument in one of the BeppoSAX observations, providing evidence for variability also in the hard X-ray range. The X-ray spectra, discussed in the context of multiwavelength observations, place some interesting constraints on the properties and location of the high-energy emitting region.
\end{abstract}

Key words. X-rays: binaries - X-rays: individuals: LS I +61 303 - X-rays: individuals: GT 0236+610

\section{Introduction}

The peculiar radio source GT $0236+610$, unambiguously associated with the B0 Ve star LS I +61 303, is unique in its high variability and periodic radio emission (Gregory \& Taylor 1978). The radio outbursts show a periodicity of about 26.5 days (Taylor \& Gregory 1982; Gregory et al. 1999) and a further modulation of both the outburst phase and outburst peak flux with a period of $\sim 1600$ days (Gregory et al. 1999), also displayed in the $\mathrm{H}_{\alpha}$ emission line (Zamanov et al. 1999).

A faint X-ray counterpart $\left(F_{2-10 \mathrm{keV}}, 6 \times 10^{-12} \mathrm{erg} \mathrm{cm}^{-2} \mathrm{~s}^{-1}\right)$ was identified by Bignami et al. (1981) with the Einstein satellite, and later observed with ROSAT (Goldoni \& Mereghetti 1995; Taylor et al. 1996), ASCA (Leahy et al. 1997), RXTE (Harrison et al. 2000; Greiner \& Rau 2001).

The hard power law X-ray spectrum extending up to $10 \mathrm{keV}$ without breaks (photon index of $\sim 1.7$, absorbing column density, $N_{\mathrm{H}}$, of $\sim 5 \times 10^{21} \mathrm{~cm}^{-2}$ ) is clearly inconsistent with low temperature plasma emission, thus excluding the Be-star as a main contribution to the X-ray emission.

The 26.5 days periodicity, reflecting the orbital motion of the system (Gregory \& Taylor 1978; Taylor \& Gregory 1982), has also been observed in the optical band (Hutchings \& Crampton 1981; Mendelson \& Mazeh 1989), in the infrared (Paredes et al. 1994), in soft X-rays (Paredes et al. 1997) and in the $\mathrm{H}_{\alpha}$ emission line (Zamanov et al. 1999).

^ Based on obervations obtained with XMM-Newton, an ESA science mission with instruments and contributions directly funded by ESA Member States and NASA.
Spectral line observations of the radio source give a distance of $2.0 \pm 0.2 \mathrm{kpc}$ (Frail \& Hjellming 1991), implying an X-ray luminosity of $10^{33} \mathrm{erg} \mathrm{s}^{-1}$. No periodic pulsations have been detected in its X-ray and radio emission. The low X-ray luminosity and the lack of iron line emission indicate that LS I +61 303 is not a classical accreting X-ray pulsar. Although this is not surprising in view of the strong radio emission (never seen from an X-ray pulsar, see e.g. Fender 2001), the low X-ray luminosity is remarkable in a source that can only be explained with the presence of a compact object.

LS I +61 303 is very likely associated with one of the most interesting unidentified gamma-ray sources near the plane of the Galaxy, the COS-B source 2CG 135+01 (Hermsen et al. 1977). Although the association between 2 CG $135+01$ and LS I +61 303 was initially weakened by the presence of another plausible counterpart in the COS-B error region (the quasar QSO 0241+622), the reduced error box determined by EGRET (Kniffen et al. 1997) is compatible only with the position of LS I +61 303.

A recent detection of variable and likely periodic very high energy gamma-ray emission above $100 \mathrm{GeV}$ has been reported by the MAGIC collaboration (Albert et al. 2006).

The strong radio outbursts suggest the presence of a compact star, although there is no direct evidence for the existence of a neutron star (no pulsations nor X-ray bursts). Two main classes of models have been proposed for LS I +61 303, involving a highly eccentric binary system with an orbital period of 26.5 days, composed of a compact star and the Be companion. 
Table 1. Summary of the XMM-Newton observations reported here. "Exp" is the nominal exposure time of a single MOS camera. $\theta_{1}$ and $\theta_{2}$ are the orbital and super-orbital phases (Gregory 2002).

\begin{tabular}{llllll}
\hline \hline OBS ID. & $\begin{array}{l}\text { Start time } \\
\text { (dy mon yr h:mn) }\end{array}$ & $\begin{array}{l}\text { End time } \\
\text { (dy mon yr h:mn) }\end{array}$ & $\begin{array}{l}\text { Exp. } \\
\text { (ks) }\end{array}$ & $\theta_{1}$ & $\theta_{2}$ \\
\hline 00 & 27 Jan. 2005 17:41 & 28 Jan. 2005 07:37 & 48.7 & 0.61 & 0.02 \\
01 & 10 Feb. 2002 11:26 & 10 Feb. 2002 12:50 & 6.4 & 0.76 & 0.37 \\
02 & 05 Feb. 2002 06:24 & 05 Feb. 2002 03:30 & 6.4 & 0.55 & 0.37 \\
03 & 21 Feb. 2002 16:02 & 21 Feb. 2002 17:26 & 7.5 & 0.18 & 0.38 \\
04 & 17 Feb. 2002 04:40 & 17 Feb. 2002 06:04 & 6.4 & 0.01 & 0.37 \\
05 & 16 Sep. 2002 02:29 & 16 Sep. 2002 04:09 & 6.4 & 0.97 & 0.50 \\
\hline
\end{tabular}

The first class of models suggests that the radio outbursts are produced by streams of relativistic particles originating in episodes of super-Eddington accretion onto the compact object (e.g., Taylor et al. 1992). The X-ray luminosity of LS I +61 303 is orders of magnitude lower than the Eddington limit. The association with the gamma-ray source $2 \mathrm{CG} 135+01$ suggests that the bulk of the energy output is shifted from X-ray to $\gamma$-ray wavelengths, but the actual mechanism responsible for this is not well understood in the context of the supercritical accretion model.

The second class of models assumes that LS I +61 303 contains a non-accreting young rapidly rotating neutron star (e.g., Maraschi \& Treves 1981; Tavani 1994). The radio outbursts are produced by energetic electrons accelerated in the shock boundary between the relativistic wind of the young pulsar and the wind of the Be star. This scenario is supported by the shockpowered emission observed by ASCA and CGRO near the periastron in a possibly similar system, the binary PSR B1259-63 (Tavani et al. 1995), which is composed by a radio pulsar (with a period of $47 \mathrm{~ms}$ ) orbiting a Be star (orbital period of $3.4 \mathrm{yr}$ ). In this case, the high-energy emission allows a sensible diagnostics of the shock emission region where the pulsar wind interacts with the circumstellar material originating from the surface of the massive star (Tavani 1994). The modulation of the radio emission might be due to the time variable geometry of a "pulsar cavity" as a function of the orbital phase.

A third way of producing X-rays has been suggested by Campana et al. (1995), as accretion onto the pulsar magnetosphere when the stellar wind ram pressure exceeds the pulsar wind pressure, but is not large enough to penetrate the pulsar magnetospheric boundary. This situation can occur in LS I +61 303 for reasonable values of the neutron star magnetic field and spin period.

An object similar to LS I +61 303 has been discovered, the microquasar LS 5039 (Paredes et al. 2000, 2005) which is subluminous in the X-ray range (even more than LS I +61 303) and, if the proposed association with the EGRET source 3EG J1824-1514 is correct, also shows the same puzzling behavior, having $L_{\gamma}>L_{\mathrm{X}}$.

Therefore, LS I +61 303 and LS 5039 could be the first two examples of a new class of X-ray binaries with powerful $\gamma$-ray emission (see also Aharonian et al. 2005 for a HESS detection of LS 5039 at energies above $250 \mathrm{GeV}$ ). In the case of LS 5039, an ejection process, probably fed by an accretion disk, is clearly proved by a VLBA map which shows bipolar jets emerging from a central core. Its high $L_{\gamma}$ is tentatively explained by inverse Compton scattering. In LS I +61 303, VLBI observations (at $5 \mathrm{GHz}$ ) show a possible jet-like elongation at milliarcseconds scales, suggesting the presence of a one-sided collimated radio jet, as found in several other X-ray binaries. A lower limit of $0.4 \mathrm{c}$ for the intrinsic velocity of the radio jet has been derived (Massi et al. 2001).
We report here the results of a deep XMM-Newton observation performed in January 2005, together with spectral analysis of archival BeppoSAX observations never reported in literature, and for completeness we studied also other five short archival XMM-Newton observations recently analysed by Chernyakova et al. (2006).

\section{Observations and data reduction}

\subsection{XMM-Newton observations}

The XMM-Newton Observatory includes three $1500 \mathrm{~cm}^{2}$ X-ray telescopes each with an European Photon Imaging Camera (EPIC) at the focus. Two of the EPIC imaging spectrometers use MOS CCDs (Turner et al. 2001) and one uses a pn CCD (Strüder et al. 2001). Behind two of the telescopes there are Reflection Grating Spectrometers (RGS, 0.35-2 keV; den Herder et al. 2001). LS I +61 303 was observed on 2005 January 27-28 for a net exposure of $48.7 \mathrm{ks}$. Data were processed using version 6.5 of the XMM-Newton Science Analysis Software (SAS). Known hot, or flickering, pixels and electronic noise were rejected using the SAS. A further cleaning was necessary because of the presence of a soft proton flare at the beginning of the observation, reducing the net good exposure times to $42.6 \mathrm{ks}$ and $30.3 \mathrm{ks}$, respectively for the EPIC MOS and the pn. EPIC MOS used Imaging Prime Partial Mode, while EPIC pn the Small Window Mode. All EPIC cameras used the medium thickness filter.

Cleaned MOS (with pattern selection from 0 to 12) and pn (patterns from 0 to 4 ) events files have been extracted, and used for the subsequent analysis with XMMSELECT. The source spectra have been extracted from a circular region with $40^{\prime \prime}$ radius, for the MOS1, MOS2 and pn separately. Background spectra have been always extracted from source free regions of the same observation. When time selected spectra have been considered, the same time selection has been applied also to the background. Spectra have always been rebinned such that at least 20 counts per bin were present and such that the energy resolution was not over-sampled by more than a factor 3 . The response matrices have been generated using the RMFGEN and ARFGEN tools implemented in the SAS 6.5. Free relative normalizations between MOS1, MOS2 and pn instruments were included in the spectral fitting. All spectral uncertainties and upper-limits are given at $90 \%$ confidence for one interesting parameter.

We also analysed other 5 much shorter XMM-Newton observations, performed in 2002. Table 1 gives a summary of the observations and the corresponding orbital $\left(\theta_{1}\right)$ and super-orbital phases $\left(\theta_{2}\right)$. The mean error on the orbital phase is \pm 0.03 . Here we adopt as time of the zero phase $\mathrm{JD}_{0}=2443366.775$, together with the updated estimates of the orbital $\left(P_{1}=26.4960 \pm\right.$ 0.0028 days) and of the super-orbital period (a periodic 


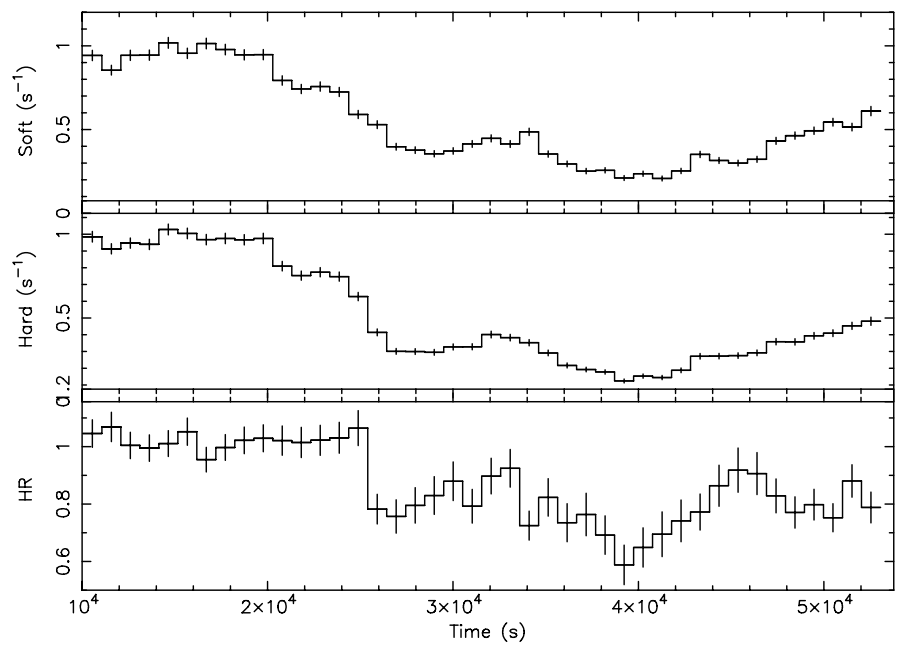

Fig. 1. Background subtracted EPIC pn lightcurves in two energy ranges (soft $=0.3-2 \mathrm{keV}$; hard $=2-12 \mathrm{keV}$ ) together with their hardness ratio $(H R=2-12 \mathrm{keV} / 0.3-2 \mathrm{keV})$. Time is in seconds starting from 2005, January 27 , at 17:56:26. Bin time is $1024 \mathrm{~s}$.

modulation of the phase and amplitude of the radio outbursts, with a period, $P_{2}$, of $1667 \pm 8$ days) reported in Gregory (2002).

\subsection{BeppoSAX observations}

LS I +61 303 was observed twice with BeppoSAX, on 1997 September 22 at 19:43 and on 1997 September 26 at 14:55 (UTC), with an on-source time of $12 \mathrm{ks}$ and $8.6 \mathrm{ks}$, respectively. We report the results obtained with the Low-Energy Concentrator Spectrometer (LECS; 0.1-10 keV; Parmar et al. 1997), the Medium-Energy Concentrator Spectrometer (MECS; 1.8-10 keV; Boella et al. 1997) and the Phoswich Detection System (PDS; 15-300 keV; Frontera et al. 1997).

Counts for the spectral analysis have been extracted from circular regions with standard radii $\left(4^{\prime}\right.$ for the MECS and $8^{\prime}$ for the LECS). The LECS and MECS spectra were rebinned to oversample the full width half maximum of the energy resolution by a factor 3 and to have a minimum of 20 counts per bin. Background spectra were extracted from annular regions around the source, in the same observations. Response matrices appropriate for the sizes of the extraction regions were used. In the spectral analysis we fitted together LECS (selected in the best calibrated energy range 0.1-4.0 keV) and MECS spectra $(1.8-10 \mathrm{keV})$, with free relative normalizations between the instruments.

The PDS consists of four independent non-imaging units arranged in pairs each having a separate collimator. Each collimator was alternatively rocked on- and $210^{\prime}$ off-source every $96 \mathrm{~s}$ during the observations. After a proper cleaning of the events files aimed at excluding the "spikes" due to single particles hits, we extracted net spectra from the two observations. Inspection and comparison of the background spectra show that there are no known contaminating sources in the background region. Since the only nearest possible high energy contaminating source is QSO 0241+622, which is located just outside the PDS field of view, at $1.4^{\circ}$, we are confident that the high energy emission is produced by LS I +61 303 .

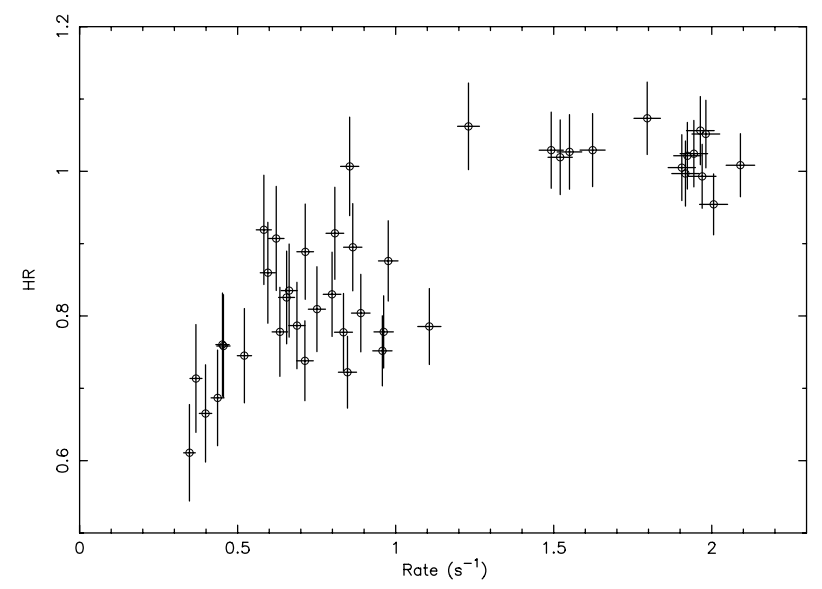

Fig. 2. Hardness ratio $(H R)$ versus intensity (count rate in the total energy range $0.3-12 \mathrm{keV}$ ) plot for the longest XMM-Newton observation (EPIC pn), with a bin time of $1024 \mathrm{~s}$. The hardness ratio is defined as ratio between net counts in the hard range $2-12 \mathrm{keV}$ and net counts in the soft energy range $0.3-2 \mathrm{keV}$.

\section{Results}

\subsection{The XMM-Newton observations}

The EPIC pn background subtracted lightcurves in two energy ranges $(0.3-2 \mathrm{keV}$ and 2-12 keV) are reported in Fig. 1, together with their hardness ratio $(H R)$. Both lightcurves show a smooth modulation, ranging from a maximum rate around 1 counts s $\mathrm{s}^{-1}$ down to about 0.2 counts $\mathrm{s}^{-1}$, in few hours. The hardness ratio shows a rather constant value of 1 for the first $15000 \mathrm{~s}$, and then a sharp decrease, where it falls down to 0.8 in less than $1000 \mathrm{~s}$. After this decrease, the hardness ratio is smoothly modulated, displaying a wave-like shape, with two maxima (around $30000 \mathrm{~s}$ and $46000 \mathrm{~s}$ in Fig. 1) and one minimum. A plot of $H R$ versus the source intensity (Fig. 2) indicates that the source is harder when it is brighter.

Since $H R$ is variable, we extracted two spectra, one corresponding to the first $14000 \mathrm{~s}$, when $H R$ was constant and the second one for the remaining part of the observation. In both cases, the continua appear featureless, and an absorbed powerlaw model gives the best-fit. There is no evidence for a break up to $12 \mathrm{keV}$, and a bremsstrahlung model is unacceptable, with a reduced $\chi^{2}$ larger than 1.4. An additional component (a blackbody) added to the power-law continuum is not formally required by the residuals, and can contribute at most $1 \%$ of the flux observed in the energy range $0.5-10 \mathrm{keV}$. Adopting a single absorbed power-law, there is no evidence for column density variations between the two time-selected spectra, thus we fixed it at $5 \times 10^{21} \mathrm{~cm}^{-2}$. The best-fit spectral results are reported in Table 2 and shown in Fig. 3.

The RGS spectra extracted from the whole observation appear featureless, with a count rate of 0.019 counts $\mathrm{s}^{-1}$ and 0.025 counts $\mathrm{s}^{-1}$ in the RGS1 and RGS2, respectively (0.3-2 keV). The combined RGS1 plus RGS2 spectra are well fitted $\left(\chi^{2} /\right.$ d.o.f. $\left.=110.7 / 127\right)$ with an absorbed powerlaw with an absorbing column density of $(6 \pm 1) \times 10^{21} \mathrm{~cm}^{-2}$, and a poorly constrained photon index of $1.9 \pm 0.6$. The statistics is too low to allow a time/hardness selected RGS spectral analysis, so we will not discuss them further.

A search for pulsations resulted in negative results.

We have reduced and analysed in a similar way also the five shorter XMM-Newton observations. Within each observation there is no evidence for significant changes in hardness 

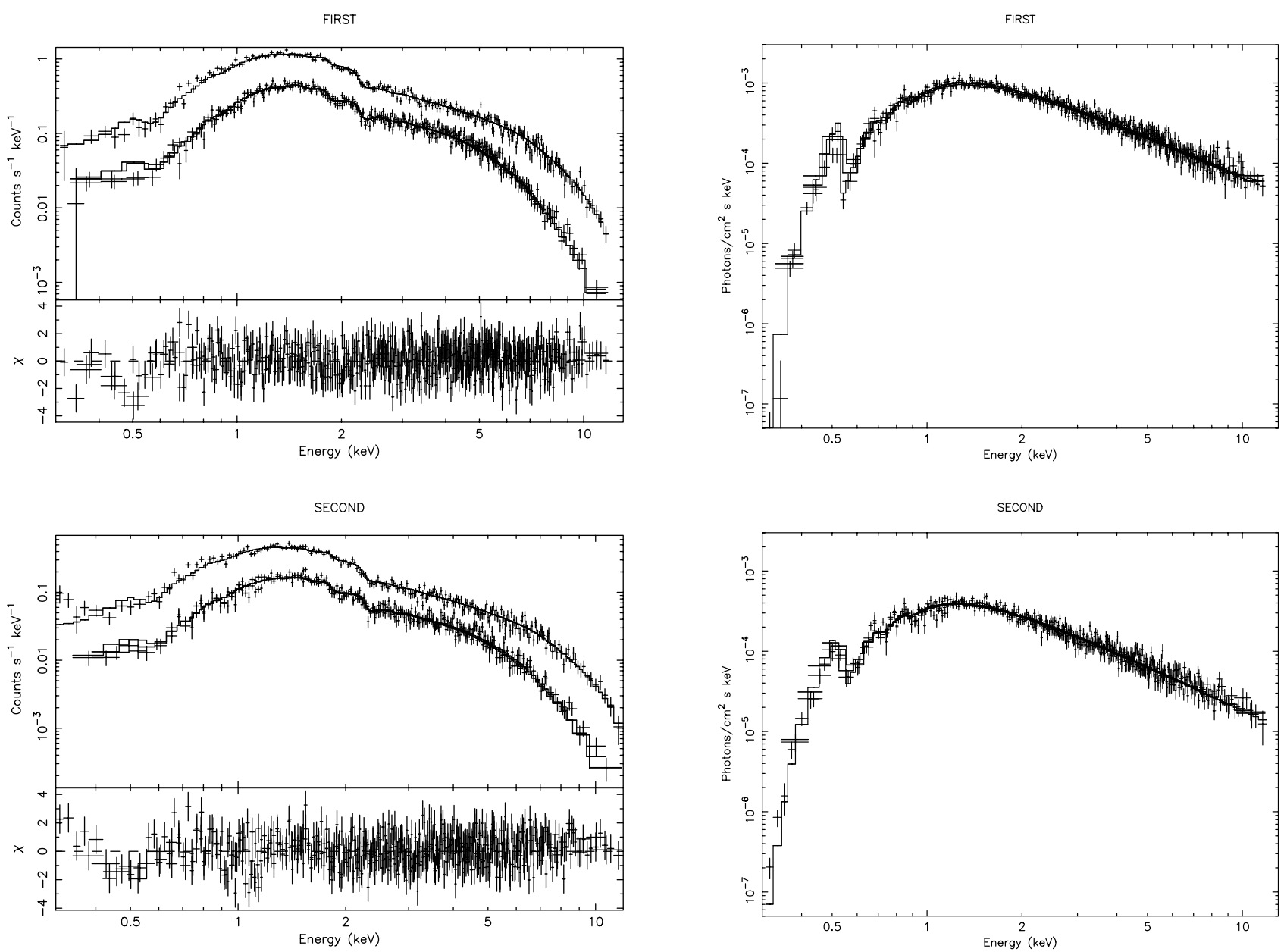

Fig. 3. Left panels: two LS I +61 303 time selected counts spectra (MOS1+MOS2+pn), together with the residuals in units of standard deviations when fitted with an absorbed powerlaw. From top to bottom, the first and the second spectra are shown. Right panels: the two corresponding time selected photon spectra.

Table 2. Best-fit results of the overall spectral analysis. X00 first and $X 00$ second indicate the two time-selected spectra (MOS1+MOS2+pn, $0.3-12 \mathrm{keV})$ of the longest XMM-Newton observation, $X 01-$ $X 05$ mark the five short XMM-Newton observations, while $S 01$ and $S 02$ indicate the results from the two BeppoSAX pointings (LECS+MECS2+MECS3; 0.1-10 keV). The best-fit model is and absorbed power-law, with the absorbing column density fixed at $5 \times$ $10^{21} \mathrm{~cm}^{-2}$. Fluxes are corrected for the absorption and are in units of $10^{-12} \mathrm{erg} \mathrm{cm}^{-2} \mathrm{~s}^{-1}$ in the energy range $2-10 \mathrm{keV}$.

\begin{tabular}{lllc}
\hline \hline Spectrum & $\begin{array}{l}\text { Photon } \\
\text { Index }\end{array}$ & Flux & $\chi^{2} /$ d.o.f. \\
\hline X00first & $1.62 \pm 0.01$ & $12.9 \pm 0.1$ & $680.7 / 596$ \\
X00second & $1.83 \pm 0.01$ & $4.0 \pm 0.1$ & $617.5 / 533$ \\
X01 & $1.53 \pm 0.02$ & $13.5 \pm 0.1$ & $494.6 / 462$ \\
X02 & $1.60 \pm 0.02$ & $13.9 \pm 0.1$ & $489.1 / 442$ \\
X03 & $1.57 \pm 0.07$ & $5.0 \pm 0.1$ & $140.1 / 174$ \\
X04 & $1.74 \pm 0.05$ & $6.8 \pm 0.1$ & $182.2 / 178$ \\
X05 & $1.52 \pm 0.02$ & $13.6 \pm 0.1$ & $497.6 / 453$ \\
S01 & $1.68_{-0.16}^{+0.12}$ & $4.8 \pm 0.1$ & $20.0 / 31$ \\
S02 & $1.56 \pm 0.09$ & $14.0 \pm 0.1$ & $60.8 / 62$ \\
\hline
\end{tabular}

ratio, so we extracted spectra from the whole exposures (after cleaning for possible contamination from proton flares). The spectral results obtained fitting the X-ray emission with an absorbed power-law (and absorbing column density fixed at $5 \times$ $10^{21} \mathrm{~cm}^{-2}$ ), are reported in Table 2 .

\subsection{BeppoSAX observations}

The background subtracted lightcurves of LS I +61 303 during the two BeppoSAX observations are shown in Fig. 4. The source was on average $\sim 2.5$ times brighter during the second observation. Within each BeppoSAX observation the source displays some evidence for flux variability, especially at the beginning of the first observation, where a sort of "flare" is present, with the count rate increasing from $\sim 0.05$ counts $\mathrm{s}^{-1}$ to $\sim 0.23$ counts $\mathrm{s}^{-1}$ during the first $1500 \mathrm{~s}$, without evidence, within the uncertainties, for a change in hardness ratio.

An absorbed power-law is the best-fit to both spectra (LECS+MECS2+MECS3), with the parameters reported in Table 2 . We fixed the absorbing column density to $5 \times 10^{21} \mathrm{~cm}^{-2}$. A bremsstrahlung model is not a good fit to the spectra, resulting in a temperature higher than $18 \mathrm{keV}$, well above the MECS spectral range. A single absorbed blackbody is unacceptable, with reduced $\chi^{2}>2$.

The analysis of the PDS data resulted in a detection at high energy (15-70 keV) only in the second observation, which indeed catched the source at a higher flux level, almost three times higher than in the first one. The PDS count rates are $0.098 \pm$ 0.048 counts $\mathrm{s}^{-1}$ and $0.31 \pm 0.07$ counts $\mathrm{s}^{-1}$, respectively for the first and second observation $(15-70 \mathrm{keV})$. Thus, only the second can be considered a detection (at $4.4 \sigma$ ). This PDS rate translates into a $15-70 \mathrm{keV}$ flux of about $3 \times 10^{-11} \mathrm{erg} \mathrm{cm}^{-2} \mathrm{~s}^{-1}$, adopting the best-fit reported in Table 2 . 

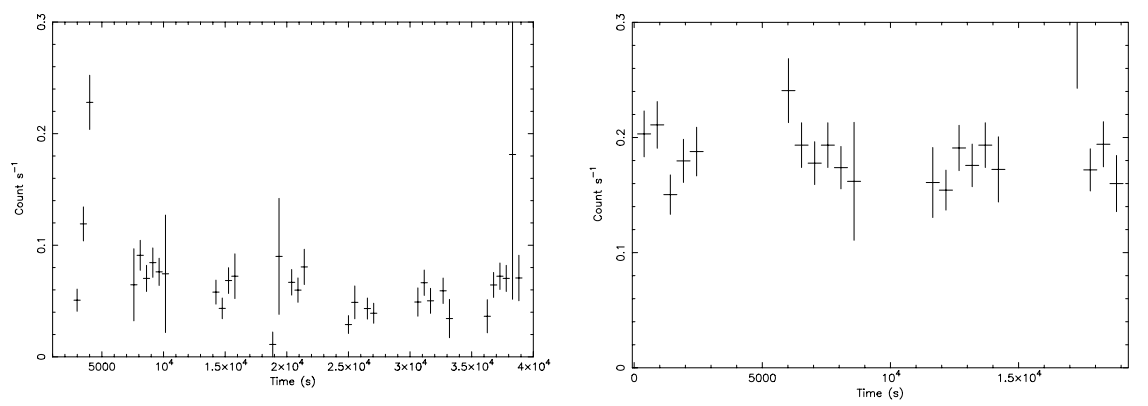

Fig. 4. The BeppoSAX MECS2+MECS3 background subtracted lightcurves during the two observations (on the left and on the right, respectively). The bin size is $512 \mathrm{~s}$. A large flux variability is visible at the beginning of the first BeppoSAX observation, with no evidence for simultaneous hardness ratio variations.
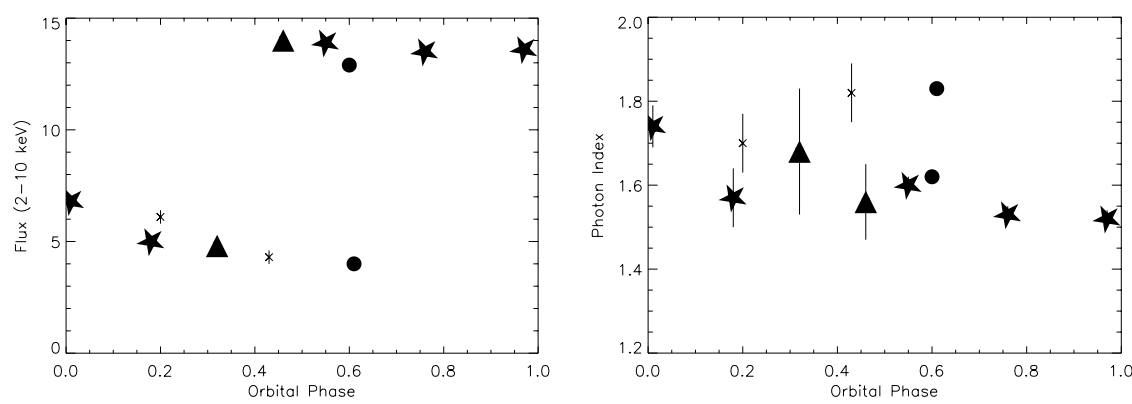

Fig. 5. Summary of the dependence with the orbital phase of the X-ray spectral parameters of all the observations analysed here (six XMM-Newton and two BeppoSAX observations) together with the ASCA results (published by Leahy et al. 1997) for completeness. The meaning of the symbols is as follows: thick circles represent the longest XMM-Newton observation, splitted into two (the "first" and the "second" spectra, with significantly different hardness ratio and flux); the other 5 XMM-Newton observations are marked with thick stars, the 2 BeppoSAX observations are indicated with thick triangles and the 2 ASCA observations (from Leahy et al. 1997) with thin crosses. Fluxes are corrected for the absorption and in units of $10^{-12} \mathrm{erg} \mathrm{cm}^{-2} \mathrm{~s}^{-1}$.

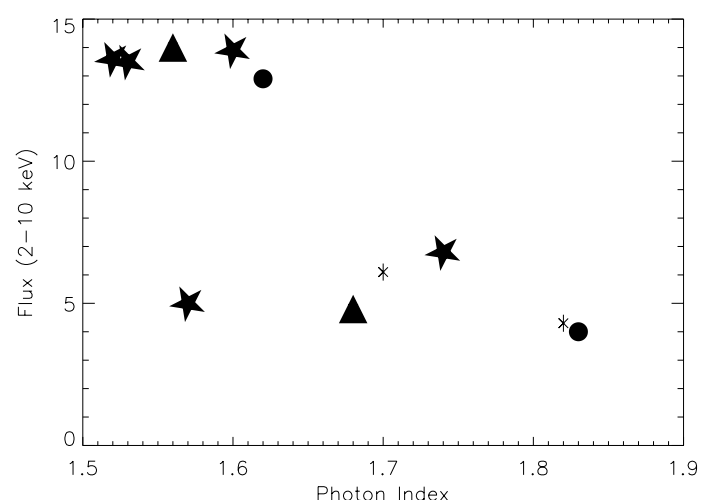

Fig. 6. Unabsorbed flux (2-10 keV; in units of $\left.10^{-12} \mathrm{erg} \mathrm{cm}^{-2} \mathrm{~s}^{-1}\right)$ versus power-law photon index, collecting all X-ray observations analysed here, together with the ASCA results (published by Leahy et al. 1997). The meaning of the symbols is the same as in Fig. 5. The source appears to be softer when it is fainter.

\section{Discussion and conclusions}

We have reported here the deepest X-ray observation of LS I +61 303 ever carried out. A systematic analysis of other five XMM-Newton and two BeppoSAX observations has also been performed, in order to investigate possible changes of the spectral parameters along the orbital phase (see Figs. 5 and 6) and to get a global picture at X-rays.

During the longest XMM-Newton observation for the first time we found evidence for a rapid (on timescales of few hours) change in flux and hardness ratio: the source flux decreased by a factor of $\sim 3$, together with a drop in hardness ratio (see Fig. 1) within about $1000 \mathrm{~s}$.
This kind of variability (the source is harder when is brighter) is also visible when comparing different observations performed in different times and with different satellites (Fig. 6). Another interesting variability feature is the presence of a sort of "flaring" behaviour at the beginning of one of the BeppoSAX observations, but with no evidence for simultaneous hardness variations, mainly because of the low statistics.

The evolution of the source X-ray flux with the orbital phase shows X-ray emission along all the phases, although with a difference of a factor 3 in the flux level between two states, with a "high" state preferentially found in the phase range 0.4-1.0. We found no obvious correlation of any of the X-ray spectral parameters with the superorbital period.

The analysis of archival BeppoSAX observations allowed the first detection of the source with the PDS instrument (in the energy range 15-70 keV), with evidence for a change in flux between a high and low state also in the hard energy band.

The observations reported here demonstrate that the source is variable both at soft and hard X-rays, also at short timescales. Thus, a detailed study of the overall spectrum should be in principle performed with simultaneous observations at all wavelengths. We try here to discuss the broad band energy spectrum distinguishing at least two source states, a high and a low state, in order to get informations on the source energetics.

Figure 7 shows our XMM-Newton and BeppoSAX results in the multiwavelength spectral energy distribution (SED) of LS I +61 303. For radio, XMM-Newton and BeppoSAX data we show both high and low state spectra. CGRO and MAGIC observations show low significance flux variations (peaking in the phase interval 0.4-0.7) correlated with those at X-ray energies. For these instruments we plot only average measurements of the datasets with positive detections. During the high 


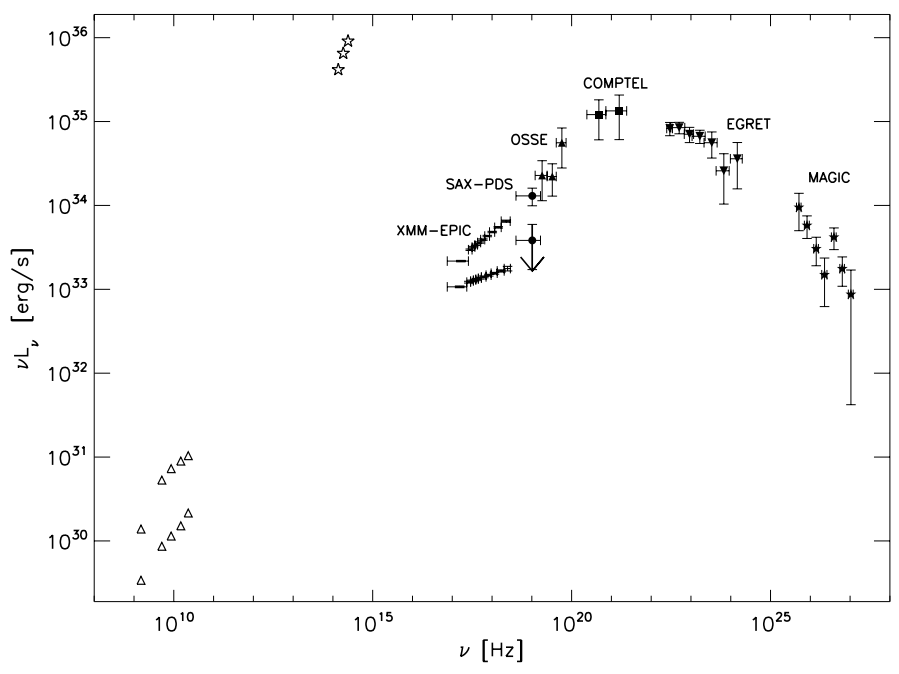

Fig. 7. Broad band LS I +61 303 spectrum from radio wavelengths to $\mathrm{TeV}$ energies. Radio data for high and low states (open triangles), IR data (open stars) and OSSE data (filled triangles) are from Strickman et al. (1998). XMM-EPIC data (crosses) and SAX-PDS data (filled circles) both high and low states are from this work. COMPTEL data (filled squares) are from van Dijk et al. (1996). EGRET data (upsidedown filled triangles) are from Kniffen et al. (1997). MAGIC data (filled stars) are from Albert et al. (2006).

state, the keV-MeV spectrum can be fit with a hardening power law $(\Gamma=1.6-1.5)$ up to the break in the COMPTEL energy range $(\sim 1-10 \mathrm{MeV})$. Then the spectrum shows a shallow rolloff (EGRET: $\Gamma=2.1$; MAGIC: $\Gamma=2.6$ ) extending up to TeV energies.

Two main models to account for the SED of LS I +61 303 have been proposed. The "micro-quasar" model involves streams of relativistic particles originating in episodes of superEddington accretion onto a compact star embedded in the mass outflow of the B-star (Taylor \& Gregory 1984; Massi et al. 2004; Massi 2004). Alternatively LS I +61 303 might contain a nonaccreting young pulsar in orbit around the mass-losing B-star (Maraschi \& Treves 1981; Dubus 2006). In both cases, the high energy emission offers a diagnostics of the shock emission parameters in the jet or in the region where the hypothetical pulsar wind would interact with the circumstellar material.

The spectral index and break of the keV-MeV emission is consistent with that of diffusive shock acceleration models in the "slow cooling" regime (i.e. far from energetic equilibrium between particle injection and cooling, see e.g. Chevalier 2000). The shallow roll-off in the $\mathrm{MeV}-\mathrm{TeV}$ energy range requires further model components in addition to the synchtrotron emission from shock-accelerated particles. However, the large error bars of the observations in this energy range can hardly constrain the several possibile contributions based both on leptonic mechanisms as Compton-scattered stellar radiation, synchrotron selfCompton, pair cascades (Dubus 2006; Dermer \& Böttcher 2006; Bosch-Ramon et al. 2006; Bednarek 2006), and on hadronic mechanisms as proton interactions, producing gamma-rays via neutral pion decay (Romero et al. 2003, 2005).

A clear signature of the SED is instead the spectral break in the $1-10 \mathrm{MeV}$ range, possibly related to the maximum energy achievable from electrons in the shock acceleration accounting for synchrotron and Compton-scattered stellar radiation energy losses, the two major electron cooling mechanisms in the dense
LS I +61 303 environment. The maximum Lorentz factor of shocked particle is obtained by solving the equation:

$\frac{1}{t_{\mathrm{acc}}}=\frac{1}{t_{\mathrm{synch}}}+\frac{1}{t_{\mathrm{comp}}}$

where $t_{\mathrm{acc}}=\xi t_{\mathrm{gyr}}=m_{\mathrm{e}} c \gamma \xi / e B$ is the electron acceleration time to reach a Lorentz factor $\gamma$ in a magnetic field with mean intensity $B$, and $\xi \gtrsim 1$ because the acceleration time in Fermi processes cannot be smaller than the gyration time, i.e. an electron gains at most a fraction of its energy when executing a single gyration in first-order shock or second-order stochastic Fermi processes (see e.g. Dermer $\&$ Böttcher 2006). $t_{\text {synch }}=$ $6 \pi m_{\mathrm{e}} c / \sigma_{\mathrm{T}} B^{2} \gamma$ is the synchrotron cooling time for an electron with Lorentz factor $\gamma$, and $t_{\text {comp }}$ accounts for inverse Compton energy losses. The solution of Eq. (1) is:

$$
\begin{aligned}
\gamma_{\max } & =\sqrt{\frac{6 \pi e}{\sigma_{\mathrm{T}} \xi B\left(1+t_{\mathrm{synch}} / t_{\mathrm{comp}}\right)}} \\
& =\frac{1.2 \times 10^{8}}{\sqrt{\xi B[\mathrm{G}]\left(1+t_{\mathrm{synch}} / t_{\mathrm{comp}}\right)}} .
\end{aligned}
$$

If $t_{\text {synch }} \ll t_{\text {comp }}$ then the maximum synchrotron photon energy does not depend on the magnetic field $\mathrm{B}$ : $h v_{\max }=$ $\hbar e B \delta \gamma_{\max }^{2} / m_{\mathrm{e}} c \simeq 167 \delta / \xi \mathrm{MeV}$, where $\delta$ is the Doppler factor to be included for jet scenarios $(1<\delta<2$, Chernyakova et al. 2006). Thus, neglecting inverse Compton losses, the spectral break frequency would be more than one order of magnitude higher than that observed.

Since photons from the Be star have a mean energy of $2.7 k_{\mathrm{B}} T \simeq 6.5 \mathrm{eV}\left(T_{\mathrm{s}}=2.8 \times 10^{4} \mathrm{~K}\right)$, the turning point between Thompson and Klein-Nishina inverse Compton regime is for $\gamma_{\mathrm{KN}}=m_{\mathrm{e}} c^{2} / h v \simeq 8 \times 10^{4}$. Even in case of severe inverse Compton energy losses, Eq. (2) shows that $\gamma_{\max }$ cannot be lower than $\gamma_{\mathrm{KN}}$ for typical magnetic fields values expected in microquasar jets or pulsar-stellar winds shocks (up to several Gauss). Thus $t_{\text {comp }}=t_{\mathrm{KN}}$ and considering electrons with Lorentz factor $\gamma$ interacting with a blackbody surface radiation field (Blumenthal \& Gould 1970):

$$
\begin{aligned}
t_{\mathrm{KN}} & =\gamma\left(\frac{r}{R_{\mathrm{s}}}\right)^{2} \frac{64 \lambda_{\mathrm{C}}^{3}}{8 \pi^{3} c \sigma_{\mathrm{T}} \Delta^{2}}\left(\frac{m_{\mathrm{e}} c^{2}}{k_{\mathrm{B}} T_{\mathrm{s}}}\right)^{2}\left[\ln \left(0.552 \Delta \gamma \frac{k_{\mathrm{B}} T_{\mathrm{s}}}{m_{\mathrm{e}} c^{2}}\right)\right]^{-1} \\
& \approx 10^{-30} \gamma r[\mathrm{~cm}]^{2} \mathrm{~s}
\end{aligned}
$$

where $R_{\mathrm{S}}=13.4 R_{\odot}$ is the Be star radius, $r$ is the distance between the star and the shock region and $\Delta \lesssim 1$ accounts for the Lorentz boosting of the stellar radiation field into the co-moving frame of the emission region (in the microquasar scenario).

Substituting Eq. (3) in Eq. (2) we obtain a general expression for the maximum Lorentz factor of the electrons accounting both for synchrotron and inverse Compton losses in the $\mathrm{KN}$ regime:

$\gamma_{\max } \simeq \sqrt{\frac{1.44 \times 10^{16} B[\mathrm{G}]-8 \times 10^{38} \xi r[\mathrm{~cm}]^{-2}}{\xi B[\mathrm{G}]^{2}}}$

which corresponds to a break energy of

$h v_{\max }=\delta\left(\frac{167}{\xi}-\frac{10^{25}}{B[\mathrm{G}] r[\mathrm{~cm}]^{2}}\right) \mathrm{MeV}$

The observed break energy at 1-10 MeV constrains the value of $B r^{2} \approx 10^{23} \mathrm{G} \mathrm{cm}^{2}$. Shock models associated to pulsar wind interacting with the circumstellar environments predict a standoff distance of the order of $r \approx 10^{11} \mathrm{~cm}$ and magnetic fields of 
a few Gauss (Dubus 2006) in good agreement with the above constraint.

On the other hand, in accreting models, a shock originated in an extended jet $\left(r \approx 10^{14}-10^{15} \mathrm{~cm}\right.$; Massi et al. 2001, 2004) would imply unrealistic low values for the magnetic fields, unless other cooling processes apart from inverse Compton on stellar radiation could play a major role (e.g. SSC, Gupta \& Böttcher 2006). Shocks originating in the inner jet at a distance comparable with the compact object-star separation $\left(r \approx 10^{12} \mathrm{~cm}\right)$ would imply more realistic values for the magnetic fields.

Future deeper and simultaneous observations in the $\mathrm{MeV}-\mathrm{GeV}$ energy range with AGILE and GLAST satellites and in VHE gamma-rays with MAGIC and HESS telescopes will better assess the IC parameters (and/or hadronic mechanisms) and thus the actual emission region properties.

Acknowledgements. The XMM-Newton data analysis is supported by the Italian Space Agency (ASI), through contract ASI/INAF I/023/05/0.

\section{References}

Albert, J., Aliu, E., Anderhub, H., et al. 2006, Science, 312, 1771 Apparao, K. M. V. 2001, A\&A, 366, 865

Aharonian, F., Akhperjanian, A. G., Aye, K. M., et al. 2005, Science, 309, 746 Bednarek, W. 2006, MNRAS, 371, 1737

Bignami, G. F. B., Caraveo, P. A., Lamb, R. C., Markert, T. H., \& Paul, J. A. 1981, ApJ Lett., 247, L85

Blumenthal, G. R., \& Gould, R. J. 1970, Rev. Mod. Phys., 42, 237

Boella, G., Chiappetti, L., Conti, G., et al. 1997, A\&AS, 122, 327

Bosch-Ramon, V., Romero, G. E., \& Paredes, J. M. 2006, A\&A, 447, 263

Campana, S., Stella, L., Mereghetti, S., \& Colpi, M. 1995, 297, 385

Chernyakova, M., Neronov, A., \& Walter, R. 2006, MNRAS, in press [arXiv: astro-ph/0606070]

Chevalier, R. A. 2000, ApJ, 539, L45

Den Herder, J. W., Brinkman, A. C., Kahn, S. M., et al. 2001, A\&A, 365, L7

Dermer, C. D., \& Böttcher, M. 2006, ApJ, 643, 1081

Dickey, J. M., \& Lockman, F. J. 1990, ARA\&A, 28, 215
Dubus, G. 2006, A\&A, 456, 801

Fender, R. 2001, in Relativistic flows in Astrophysics, Springer Verlag Lecture Notes in Physics, ed. A. W. Guthmann, M. Georganopoulos, K. Manolakou, \& A. Marcowith

Frail, D. A., \& Hjellming, R. M. 1991, AJ, 101, 2126

Frontera, F., Costa, E., Dal Fiume, D., et al. 1997, A\&AS, 122, 371

Goldoni, P., \& Mereghetti, S. 1995, A\&A, 299, 751

Gregory, P. C. 2002, ApJ, 575, 427

Gregory, P. C., \& Taylor, A. R. 1978, Nature, 272, 704

Gregory, P. C., Peracaula, M., \& Taylor, A. R. 1999, ApJ, 520, 376

Greiner, J., \& Rau, A. 2001, A\&A, 375, 145

Gupta, S., \& Böttcher, M. 2006, ApJ, submitted [arXiv: astro-ph/0606590]

Harrison, F. A., Ray, P. S., Leahy, D. A., et al. 2001, ApJ, 528, 454

Hermsen, W., Swanenburg, B. N., Bignami, G. F., et al. 1977, Nature, 269, 494

Hutchings, J. B., \& Crampton, D. 1981, PASP, 93, 486

Kniffen, D. A., Alberts, W. C. K., Bertsch, D. L., et al. 1997, ApJ, 486, 126

Leahy, D. A., Harrison, F. A., \& Yoshida, A. 1997, ApJ, 475, 823

Maraschi, L., \& Treves, A. 1981, MNRAS, 194, 18

Massi, M. 2004, A\&A, 422, 267

Massi, M., Ribó, M., Paredes, J. M., et al. 2001, A\&A, 376, 217

Massi, M., Ribó, M., Paredes, J. M., et al. 2004, A\&A, 414, L1

Mendelson, H., \& Mazeh, T. 1989, MNRAS, 239, 733

Paredes, J. M., Estallela, R., \& Ruis, A. 1990, A\&A, 232, 337

Paredes, J. M., Marziani, P., Martí, J., et al. 1994, A\&A, 288, 519

Paredes, J. M., Martí, J., Peracaula, M., \& Ribó, M. 1997, A\&A, 320, L25

Paredes, J. M., Martí, J., Ribó, M., \& Massi, M. 2000, Science, 288, 2340

Paredes, J. M., Bosch-Ramon, V., \& Romero, G. E. 2005, A\&A, 451, 259

Parmar, A. N., Martin, D. D. E., Bavdaz, M., et al. 1997, A\&AS, 122, 309

Romero, G. E., Torres, D. F., Kaufman Bernadó, M. M., \& Mirabel, I. F. 2003, A\&A, 410, L1

Romero, G. E., Christiansen, H. R., \& Orellana, M. 2005, ApJ, 632, 1093

Strickman, M. S., Tavani, M., Coe, M. J., et al. 1998, ApJ, 497, 419

Strüder, L., Briel, U., Dennerl, K., et al. 2001, A\&A, 365, L18

Taylor, A. R., \& Gregory, P. C. 1982, ApJ, 255, 210

Taylor, A. R., \& Gregory, P. C. 1984, ApJ, 283, 273

Taylor, A. R., Kenny, H. T., Spencer, R. E., \& Tzioumis, A. 1992, ApJ, 395, 268

Taylor, A. R., Young, G., Peracaula, M., et al. 1996, A\&A, 305, 817

Turner, M. J. L., Abbey, A., Arnaud, M., et al. 2001, A\&A, 365, L27

van Dijk, R., Bennet, K., Bloemen, H., et al. 1996, A\&A, 315, 485

Zamanov, R. K., Martí, J., Paredes, J. M., et al. 1999, A\&A, 351, 543 